\title{
Quality assurance and quality control in environmental radioactivity monitoring
}

\author{
J.D. Krneta Nikolić", D.J. Todorović, M.M. Janković, G.K. Pantelić and M.M. Rajačić \\ University of Belgrade Vinča Institute of Nuclear Sciences, P.O. Box 522, 11001 Belgrade, Serbia; jnikolic@vinca.rs
}

Received: 17 December 2012 / Accepted: 16 June 2013

(c) 2014 Wageningen Academic Publishers

\section{RESEARCH ARTICLE}

\begin{abstract}
To assure that radiological monitoring is reasonably valid, organisations performing such measurements have found it necessary to establish quality assurance (QA) and quality control (QC) programs. These programs are needed to identify deficiencies in the sampling and measurement processes. In addition, validation of the monitoring aims to prove whether the results are acceptable. The major aim of QA and QC in analytical laboratories is to address measures used to verify the validity of the final results. In the document ISO 17025 the requirements on how to achieve QA and QC in analytical laboratories is described. All proposed measures for QC/QA are implemented in the Radiation and Environment Protection Department of Institute for Nuclear Sciences in Vinča, Belgrade. This paper presents the summarisation and analysis of QC and QA measures undertaken by the Laboratory for Environmental and Radiation Protection of the Vinča Institute of Nuclear Sciences. Total of 6 months of QC/QA charts are presented in this paper for 2 HPGe gamma spectrometry detectors and one gross alpha/beta detector. The data were analysed and conclusions were made concerning the stability of the measurement systems. In cases of unsatisfactory behaviour of the system, some correctional measures are proposed and conducted and the effect on the measurement system is discussed.
\end{abstract}

Keywords: environmental monitoring, quality control, quality assurance

\section{Introduction}

Analyses of radionuclides in environmental samples are carried out daily in many laboratories. These data are used for a variety of purposes including environmental surveys (Popović et al., 2007) and dose assessment in man and the environment, the establishment of standards of regulatory guides (Poms and Astley, 2011), and in decision making on economic, legal and health aspects (Betti and Aldave de las Heras, 2004). It is important to assure the accuracy and precision of the analysis results to guarantee that decisions are based on reliable results. The increasing need for reliable data creates a concomitant need for a quality assurance (QA) system to support the acquisition of precise, accurate data. Objective evidence of precision and accuracy are a key component of data defensibility, and critical to the success of any environmental programs which rely on analytical data for decision making (Betti and Aldave de las Heras, 2004). For that purpose, an effective quality control (QC) and QA program is necessary to maintain high quality of results.
QC ought to be planned, described in the QC documentation, performed in a systematic manner, recorded and reviewed. To reduce the fraction of results that has to be rejected, QC must be embedded into an overall systematic approach to avoid mistakes before they are made, and this is commonly referred to as 'quality assurance' (IAEA, 2004; ISO, 2006). Planning should identify and define type and frequency of QC, acceptance limits, actions if those limits are exceeded and periodic review of results.

There are several methods used for QC. Total background count rate without a source is used to verify that the detector and shield have not been contaminated by radioactive materials. The intensity and stability of the background spectrum are dependent on many factors such as material and technical characteristic of the detector, design and materials of the shielding, ventilation, etc. Any deviation of the background total count or count rate can point to possible contamination of the detector or the environment. 
Measurement of the total activity of calibration source is used to check the efficiency calibration and general operating parameters of the system (source positioning, contamination, library values, and energy calibration). The detector-shield background, detector efficiency, peak shape and peak drift are measured and verified if they are within the acceptance limits.

Also, an external control can be implemented in a form of various interlaboratory proficiency tests and intercomparisons. The analysis of the results achieved in the proficiency tests and control charts of the laboratory z-score give participants the possibility of a long term follow-up of their performance promoting the improvement of QC (Sorbo et al., 2013).

A QA program includes several steps. Among these, few can be mentioned: (a) the selection and validation of analytical methodology; (b) the resources used for the analysis (qualified personnel, work area, instrumentation and equipment, consumables, supplies, etc.); (c) laboratory operations for sample handling and analysis (reception, recording samples, data handling, reporting, archiving of results, etc.); and (d) QC, monitoring and auditing.

All of these proposed measures for QC/QA are implemented in the Radiation and Environment Protection Department of the Institute for Nuclear Sciences in Vinča, Belgrade. Since the operation of this department includes daily measurements of a large number of samples (such as building material, imported and exported goods, air filters, soil, water, etc.), it is essential to have a stable and accurate measuring system, so that the results are accurate, precise and repeatable. QC/QA procedures are implemented on weekly basis for gross alpha/beta and gamma activity measurements. Gas proportional counter Thermo Eberline FH and two HP Germanium detectors (Canberra, Meriden, CT, USA) are readily controlled in terms of accuracy, precision and repeatability of the results, as well as the stability of the instruments. Also, the laboratory participated in a series of interlaboratory proficiency test organised by the International Atomic Energy Agency (IAEA).

The control charts resulting from these QC/QA procedures namely the Shewhart control charts, which span over a period of 6 months (September 19, 2011 - April 2, 2012), are presented in this paper.

\section{Materials and methods}

The accuracy and reproducibility of gamma spectrometry systems are verified on a weekly basis. Total background count rate is monitored to verify that the detector and shielding have not been contaminated by radioactive materials.
Energy calibration is checked in a whole region of energies before applying usual QC procedure for gamma spectrometry measurement. The total activity of calibration source is used to check the efficiency calibration and the general operating parameters of the gamma spectrometry system (source positioning, contamination, library values, and energy calibration). The detector-shield background, detector efficiency, peak shape, and peak drift are measured to ascertain whether they are within the warning and acceptance limits. For that purpose ${ }^{60} \mathrm{Co}$ point source was used. Radionuclide sources were bought from the Czech Metrological Institute which is traceable to the Bureau International des Poids et Mesures (BIPM).

Several parameters were obtained with radioactive standard material (Pantelić et al., 2009):

- total activity of all nuclide(s) for a given calibration source (decay corrected);

- average full width at half maximum (FWHM) ratio (FWHM spectrum/FWHM calibration) for a list of energies emitted by a calibration source;

- average full width at tenth maximum (FWTM) ratio (FWTM spectrum/FWTM calibration) for a list of energies emitted by a calibration source; and

- average peak shift (average of the deviation of actual peak centroids from expected library energies within a specified range).

Alpha and beta efficiencies of gas proportional counter are checked by placing ${ }^{241} \mathrm{Am}$ and ${ }^{90} \mathrm{Sr}$ point sources in each of the six measuring positions of the instrument and counted for $600 \mathrm{~s}$. Point sources were issued by Czech Metrological Institute which is traceable to BIPM. Background count rate is also checked by measuring for the duration of 3,600 s. If the values obtained during a QC procedure differ from the specified values (i.e. specified by the manufacturer), a correction procedure is implemented. The steps that are undertaken in that case depend on the problem established via $\mathrm{QC}$ procedure.

\section{Results and discussion}

A total of 6 months of QC/QA charts are presented in this paper for $2 \mathrm{HPGe}$ gamma spectrometry detectors (Canberra HPGe coaxial detector p-type, model GC 20187500 and Canberra HPGe coaxial detector n-type, model 7229N-7500-1818; Canberra Industries Inc., Meriden, CT, USA) and 1 gross alpha/beta detector (Thermo Eberline FHT 770T; Thermo Eberline LLC, Franklin, MA, USA). The values that are being observed in case of gamma spectrometry detectors are background, activity and energy of point source and FWHM and FWTM of the full energy peaks. The control charts representing the results of QC measurements for HPGe detectors are presented in Figures 1, 2 and 3. In Figure 1, the total background count over 8,192 channels for both detectors is depicted. Figure 1 represents 

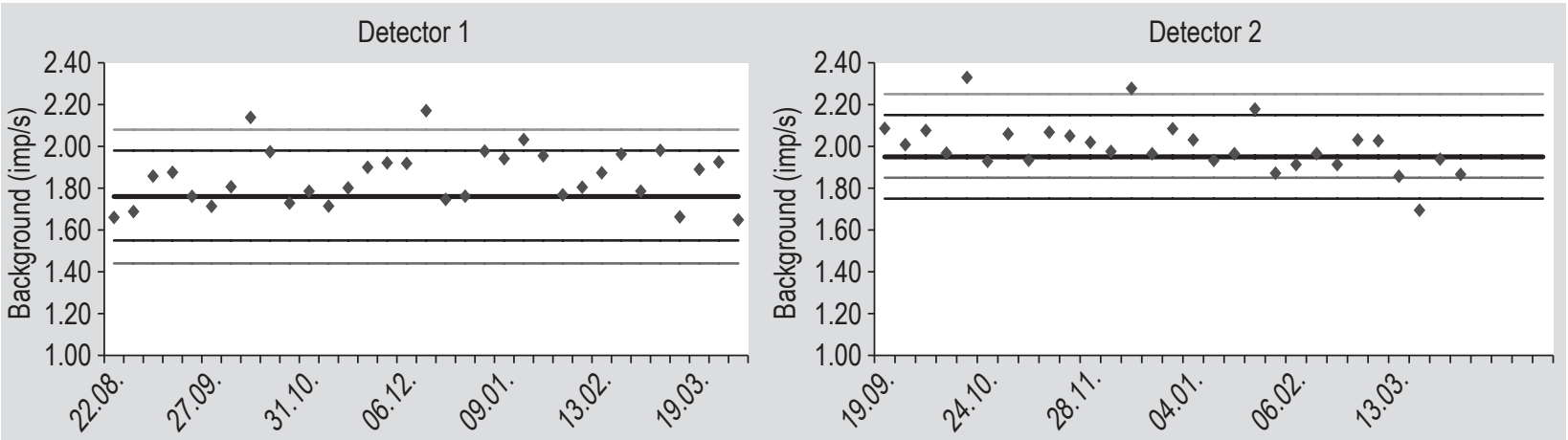

Figure 1. Total gamma background count on two HPGe gamma detectors. The acceptance limits were set at $\pm 2 \sigma$ and $\pm 3 \sigma$ around the mean value for the period.
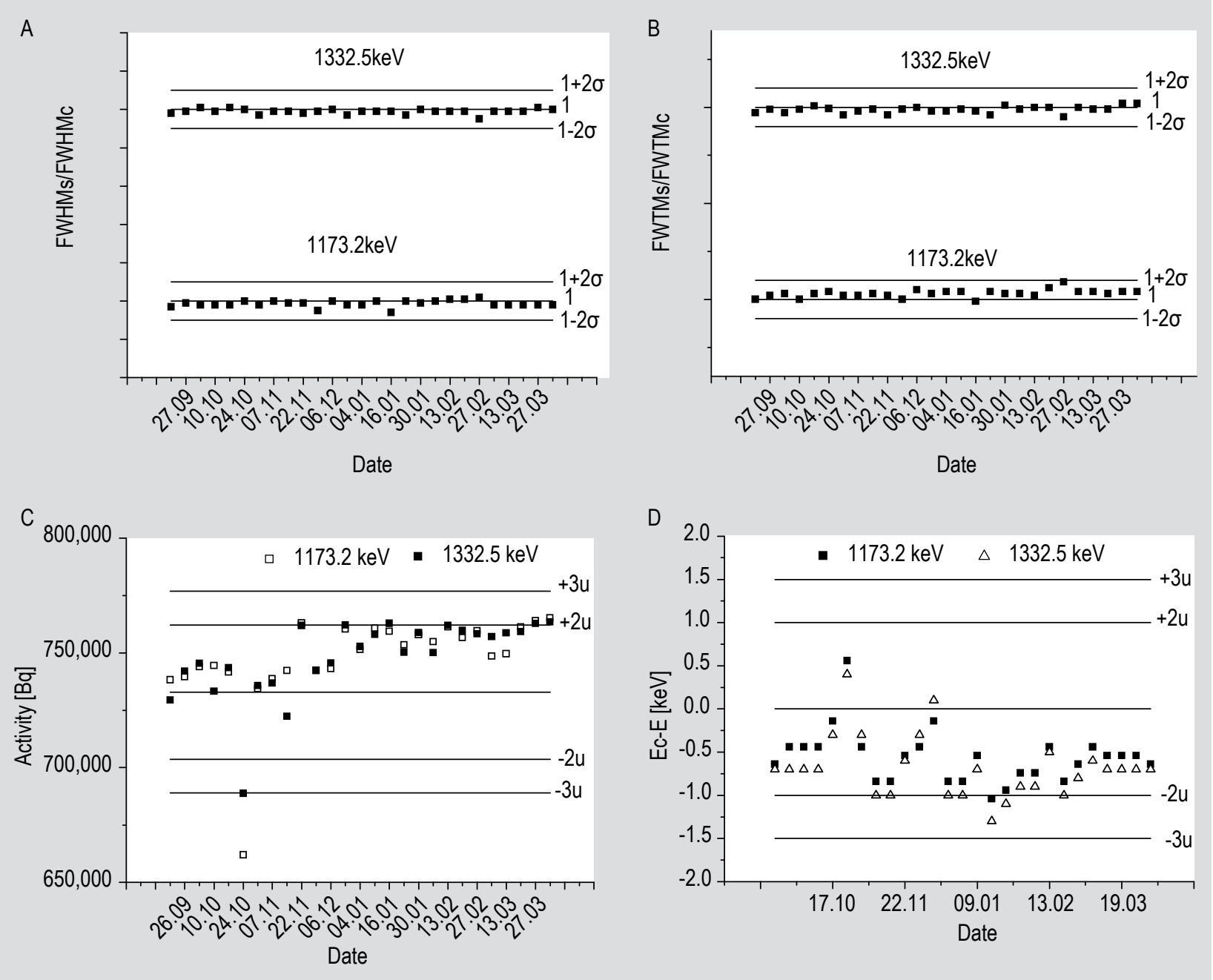

Figure 2. (A) Average full width at half maximum ratio on two energies for detector 1. FWHMs (full width at half maximum) is the measured value and FWHMc is the calibrated value. The acceptance limits are set at $\pm 2 u$ around unity. (B) Average full width at tenth maximum ratio on two energies for detector 1 . The acceptance limits are set at $\pm 2 u$ around unity. (C) Measured activity of the point source for detector 1 . The middle line represents the activity specified by the manufacturer of the point source and the acceptance limits are set to be $\pm 2 u$ and $\pm 3 u$. (D) The difference between the energy specified by the manufacturer of the point source $(E c)$ and the measured energy $(E)$ for detector 1 . The acceptance limits are set to be $\pm 2 u$ and $\pm 3 u$ around zero. 


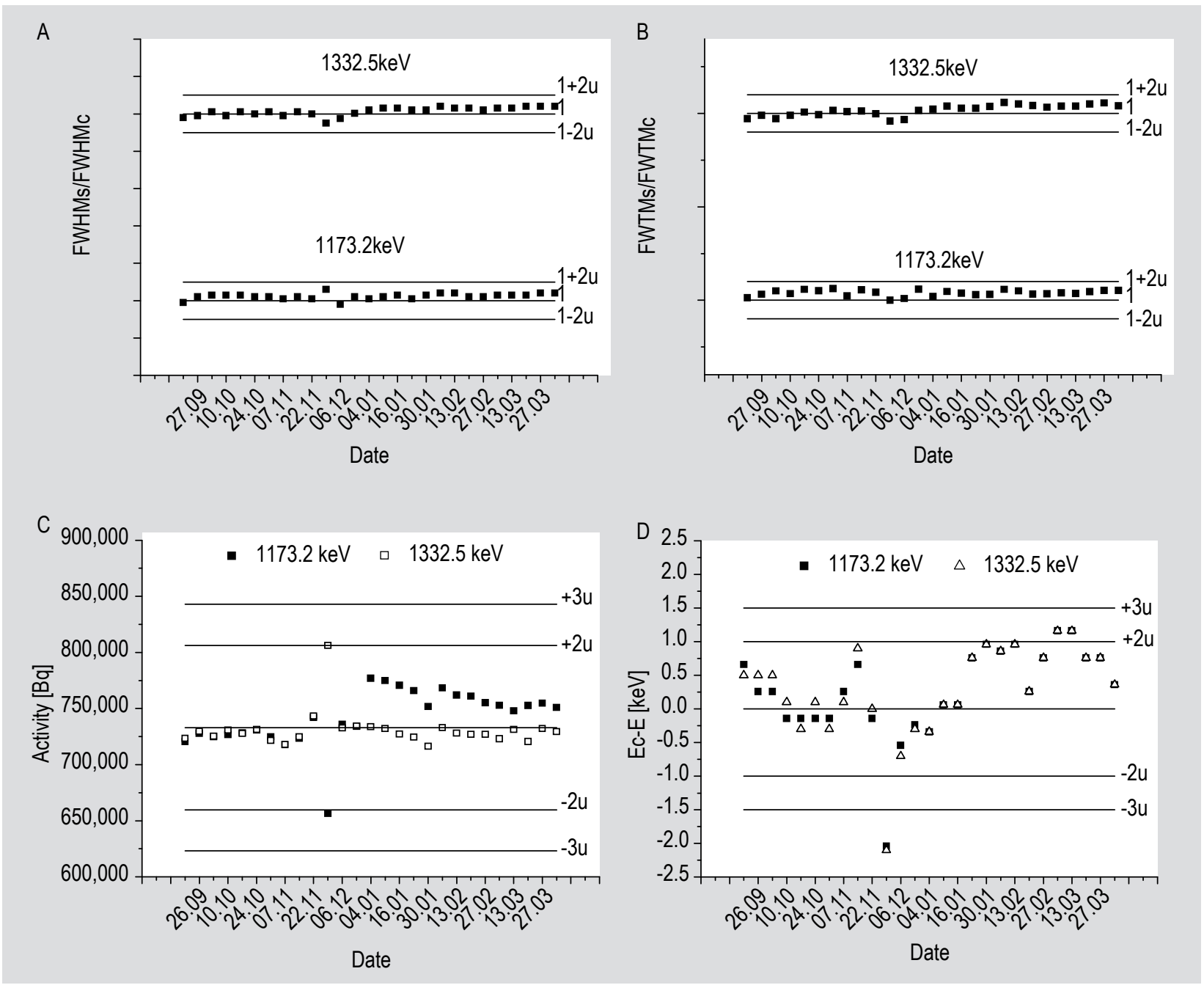

Figure 3. (A) Average full width at half maximum ratio on two energies for detector 2. FWTMs () is the measured value and FWTMc is the calibrated value. The acceptance limits are set at $\pm 2 u$ around unity. (B) Average full width at tenth maximum ratio on two energies for detector 2. The acceptance limits are set at $\pm 2 u$ around unity. (C) Measured activity of the point source for detector 2. The middle line represents the activity specified by the manufacturer of the point source and the acceptance limits are set to be $\pm 2 u$ and $\pm 3 u$. (D) The difference between the energy specified by the manufacturer of the point source (Ec) and the measured energy (E) for detector 2 . The acceptance limits are set to be $\pm 2 u$ and $\pm 3 u$ around zero.

integral background count over the time. The acceptance limits are set to be $\pm 2 \sigma$ and $\pm 3 \sigma$ around the mean value taken over a chosen period of time. Points falling between $\pm 2 \sigma$ are considered to be satisfactory, the ones inside $\pm 3 \sigma$ are warning and those exceeding $\pm 3 \sigma$ indicate that a problem in measurement of the background has occurred. As it can be seen from the figure, such cases occurred in tree occasions. As the counteraction, a thorough ventilation of the laboratory was undertaken, in order to vent the excess radon gas that built up in the room over the night. After repeated measurement, the background count was within the acceptance limits.

Figure 2 represents the QC for the first HPGe detector. The observed values are activity and energy of the point source, FWHM and FWTM. The values presented in the Figure
2 are ratio of the measured value to the value specified by the manufacturer of the detector in case of FWHM and FWTM and difference between the measured values to the value specified in the certificate of the used point source in case if energy. The ideal case for that ratio is to be a unity and zero, respectively, and the acceptance limits are set to $\pm 2 \mathrm{u}$ and $\pm 3 \mathrm{u}$, where $\mathrm{u}$ represents a combined uncertainty of the measurement, calculated according to the IAEA recommended procedure and the uncertainty of the counting, gamma emission probability and efficiency calibration (Chinnaesakki et al., 2012). While the FWHM, FWTM and the activity are in all cases within the established acceptance limits (indicating that the signal shape and amplification of the signal are in good condition, as well as the efficiency calibration), we can see that the 
peak position (centroid) is not. That indicates that the energy calibration is necessary to correct for the peak drift.

Figure 3 shows the same analysis for the second HPGe detector. In this case, the continuous decrement of the measured peak shift indicated a more serious malfunction of the system. Since FWHM, FWTM and the activity did not show any significant discrepancy, it was obvious that the problem lies within the detector itself, rather than in the preamplifier, amplifier or the high voltage supply. It was essential to perform a maintenance service for he unit in order to correct this problem which, if there were no QC measurements, would not be detected.

For gross alpha/beta proportional counter, QC background control charts are represented in Figures 4 (alpha background) and Figure 5 (beta background). As it can be seen in Figure 4, alpha background in all 6 positions of the

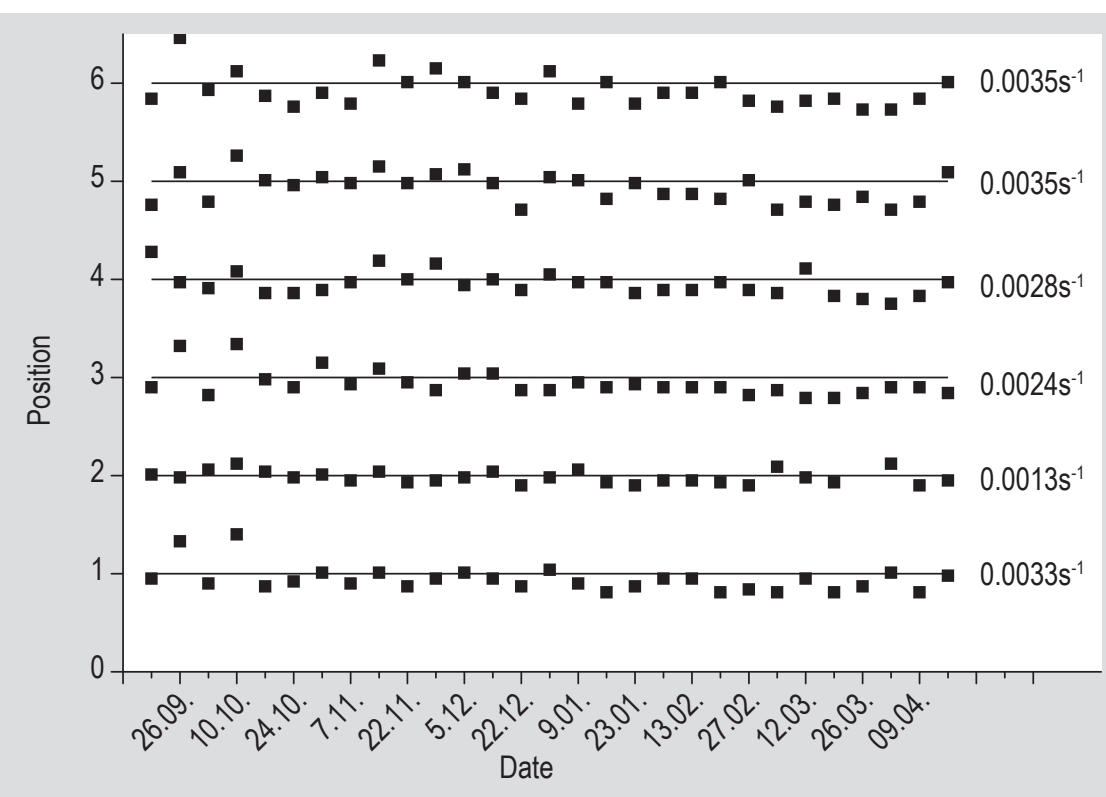

Figure 4. Total alpha background count on all 6 positions of the proportional counter. The mean value is depicted by a line on the graph and the value is indicated by the number beside the line.

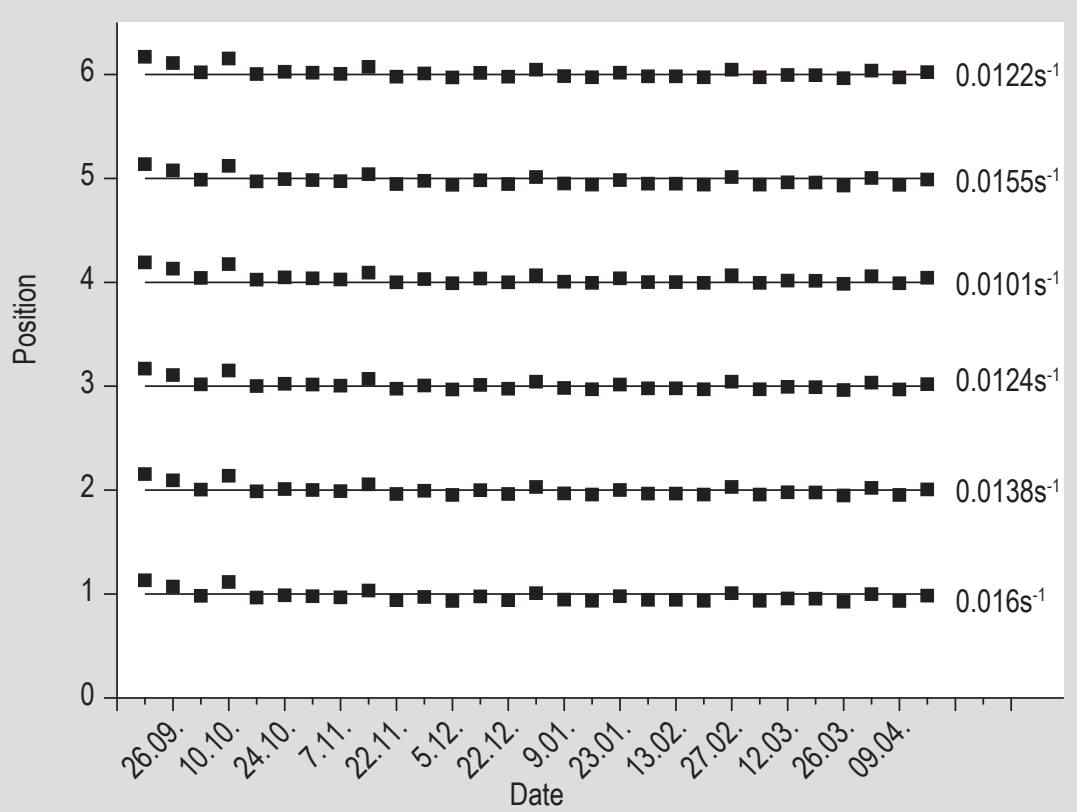

Figure 5. Total beta background count on all 6 positions of the proportional counter. The mean value is depicted by a line on the graph and the value is indicated by the number beside the line. 
proportional counter is within the established acceptance limits. This is also valid for beta background, presented in Figure 5 except for one point where background on position 6 exceeded the upper limit. In that case, the metallic planchet at that position was thoroughly cleaned with alcohol in order to remove any possible contaminants originating from the previously measured samples.
Efficiency of the alpha/beta counter was checked by placing a point source $\left({ }^{241} \mathrm{Am}\right.$ and $\left.{ }^{90} \mathrm{Sr}\right)$ in each measuring position of the counter. The control charts regarding the efficiency are presented in Figures 6 and 7. The acceptance limits are set to $\pm 2 \mathrm{u}$ and $\pm 3 \mathrm{u}$ around the value calculated according to manufacturer specified activity of the point sources, where $\mathrm{u}$ represents the combined uncertainty of the measurement. Both alpha and beta efficiencies at all positions are within

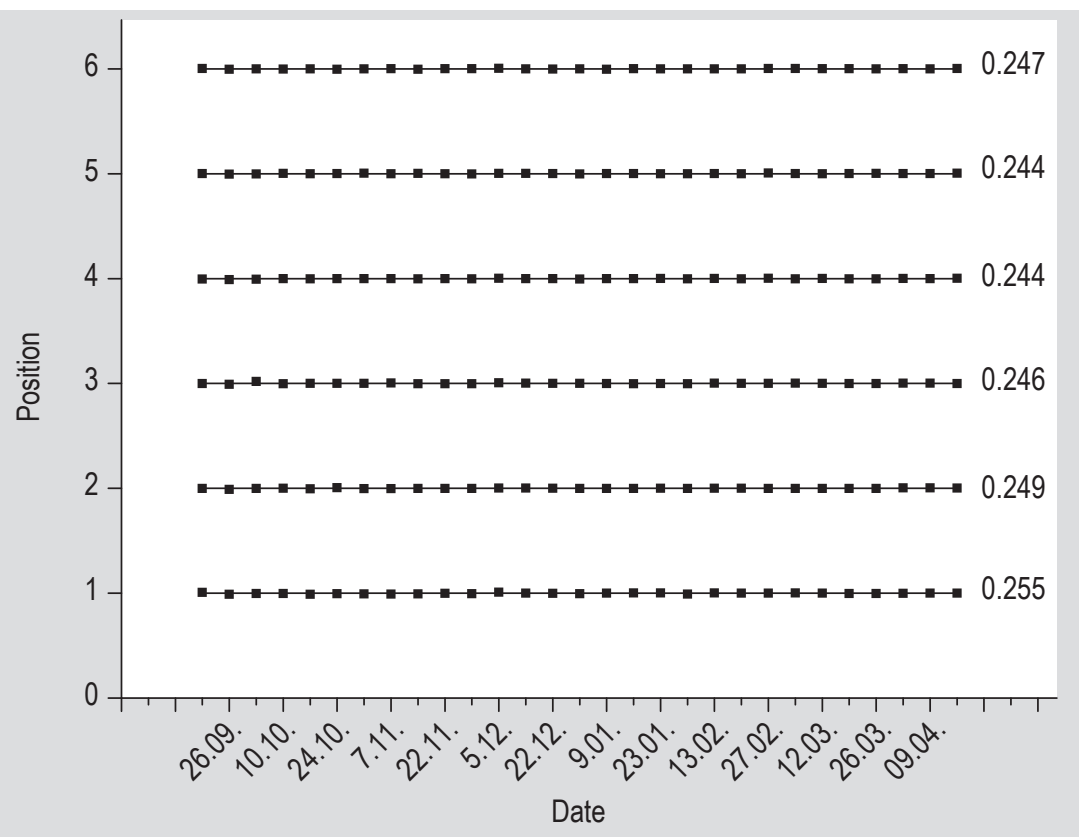

Figure 6. Alpha efficiency on all 6 positions of the proportional counter. The mean value is depicted by a line on the graph and the value is indicated by the number beside the line.

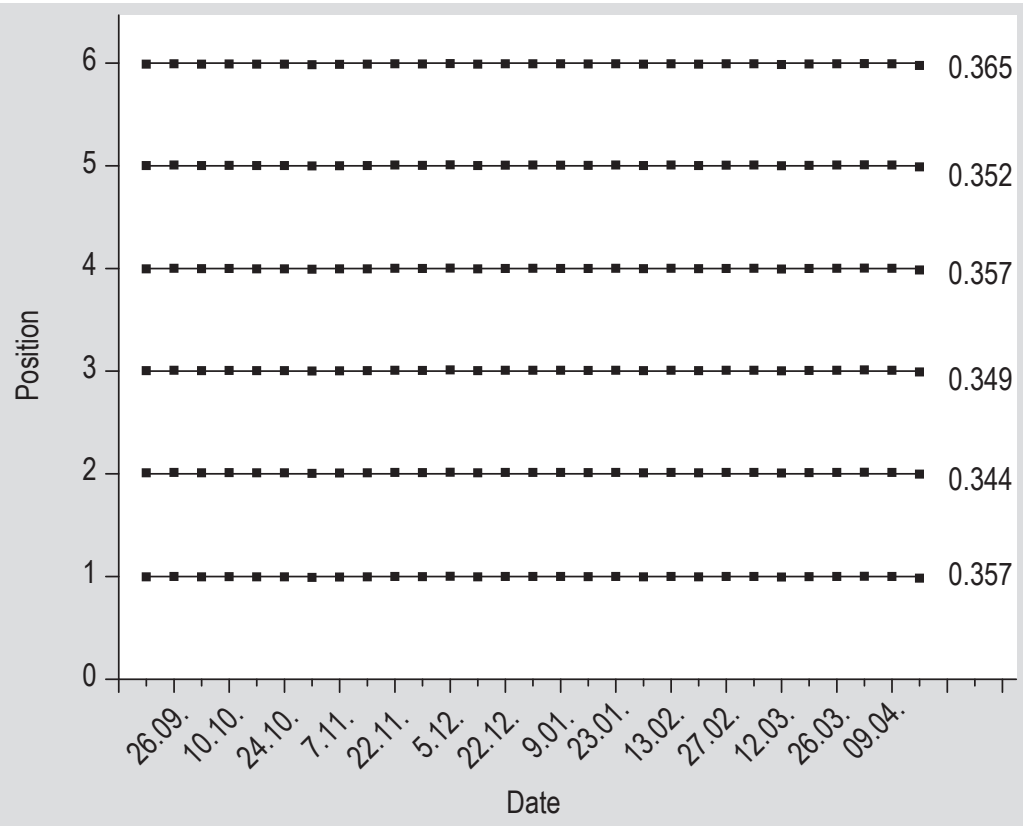

Figure 7. Beta efficiency on all 6 positions of the proportional counter. The mean value is depicted by a line on the graph and the value is indicated by the number beside the line. 
the acceptance limits except in two separate cases. However, since these discrepancies did not continue after repeated measurements, it is considered to be a statistical variation of the measurement and no specific action was performed. This shows that the gross alpha/beta proportional gas counter has a satisfactory stability, precision and accuracy.

As an additional means of QC, the Environment and Radiation Protection Department participated in a series of interlaboratory proficiency tests of the IAEA (Pantelić et $a l ., 2010)$. Here we mention only those organised in the last five years: IAEA-CU-2008-03 world-wide open proficiency test on the determination of natural radionuclides in phosphogypsum and spiked water; the IAEA-CU-2009-04 ALMERA proficiency test on the determination of radionuclides in simulated air filter; the IAEA-CU-2009-03 world-wide proficiency test on the determination of natural and artificial radionuclides in moss-soil and water; the IAEA-CU-2010-03 world-wide proficiency test on the determination of natural radionuclides in water and soil; the IAEA-CU-2010-04 ALMERA proficiency test on the determination of natural radionuclides in water and soil. The performance evaluation of the proficiency tests showed that the laboratory results were in good agreement with the target values, which served as a confirmation of the reliability and traceability of all measurements conducted in this laboratory.

\section{Conclusions}

QA and QC procedures assist the laboratory personnel to minimise potential source of errors and to obtain good measurements results (decrease rate of non-conformance results). Shewhart control charts for gross alpha/beta proportional counter and two HPGe gamma detectors, spanning 6 months of planned QC in the Radiation and Environment Protection Department, are presented in this paper. The results show a considerable stability of the measuring system and accuracy and repeatability of the measurement results. In the situations where the measured value exceeded the acceptability limits, an adequate correction procedure was implemented. As an additional means of QC, Environment and Radiation protection Department participated in a series of interlaboratory proficiency tests organised by IAEA. The evaluation results were acceptable in a considerable period of time, which serves as a confirmation of reliability of the measurements conducted in this laboratory.

\section{Acknowledgements}

The investigation was partially supported by the Ministry of Education and Science of the Republic of Serbia under project no. III43009.

\section{References}

Betti, M. and Aldave de las Heras, L., 2004. Quality assurance for the measurements and monitoring of radioactivity in the environment. Journal of Environmental Radioactivity 72: 233-243.

Chinnaesakki, S., Bara, S.V., Sartandel, S.J., Tripathi, R.M. and Puranik, V.D., 2012. Performance of HPGe gamma spectrometry system for the measurement of low level radioactivity. Journal of Radioanalytical and Nuclear Chemistry 294: 143-147.

International Atomic Energy Agency (IAEA), 2004. Quality system implementation for nuclear analytical techniques. Training course series 24. IAEA, Vienna, Austria. Available at: http://www-pub.iaea. org/MTCD/Publications/PDF/TCS-24_web.pdf.

International Organization for Standardization (ISO), 2006. General requirements for the competence of testing and calibration laboratories. ISO/IEC 17025. ISO, Geneva, Switzerland.

Pantelić, G., Vuletić, V., Eremić Savković, M., Tanasković, I. and Javorina, Lj., 2009. QA/QC in gammaspectrometry laboratory. Report of the technical meeting on quality assurance for nuclear spectrometry techniques (G4-TM-36923). October 12-16, 2009. IAEA, Vienna, Austria.

Pantelić, G., Vuletić, V. and Mitrović, R., 2010. Proficiency test of gamma spectrometry laboratories in Serbia. Applied Radiation and Isotopes 68: 1270-1272.

Poms, R.E. and Astley, S., 2011. MoniQA: an update of the European Union funded Network of Excellence in 2011. Quality Assurance and Safety of Crops and Foods 3: 89-101.

Popović, D., Todorović, D., Spasic Jokić, V. and Djurić, G., 2007. Air Radioactivity monitoring in Serbia In: Environmetal Tehnologies: New Developements, Burcu Ozkaraova Gungor, 147-166, I-Tech Inc, Vienna

Sorbo, A., Colabucci, A. and Ciaralli, L., 2013. Control charts to evaluate long-term performance in proficiency tests. Accreditation and Quality Assurance 18: 291-298. 
